\title{
The Lateral Deltoid Originates From the Entire Lateral Wall of the Acromion: MRI and Histologic Cadaveric Analysis Regarding Vertical Lateral Acromioplasty
}

\author{
Geoffrey C. S. Smith, M.B.B.Ch., F.R.A.C.S., Victor K. Liu, B.Sci. (Med), \\ S. Fiona Bonar, M.B.B.Ch., F.R.C.Path, and Patrick Hong Lam, M.D. Ph.D.
}

\begin{abstract}
Purpose: The aim of this study was to assess the nature of the middle deltoid muscle insertion onto the lateral acromion by macroscopic, MRI and histologic examination and to, therefore, assess the potential impact of a vertical lateral acromioplasty on the deltoid origin. Methods: We assessed the acromial origin of the deltoid in 6 cadaver shoulders by macroscopic, MRI and histologic examination. The cadavers were scanned with Tl and proton density-weighted sequences. H\&E- and Masson trichrome-stained histologic sections through the acromion were taken and visualized under polarized microscopy. Results: The enthesis of the deltoid muscle consisted of dense birefringent bundles of collagen that blended with the bony endplate of the acromion at all points on its lateral wall. A prominent band of collagen was seen on both MRI and histologic slices, traversing the superior surface of the acromion. It was continuous with the deltoid origin and blended with the superficial fascia of the deltoid laterally. Conclusions: The middle deltoid muscle occupies the entire lateral acromion. Clinical Relevance: A high critical shoulder angle is associated with rotator cuff tears. A lateral acromioplasty resects the lateral acromion and aims to normalize the critical shoulder angle. However, a vertical lateral acromioplasty may release the middle deltoid origin from the lateral acromion. The superior band of collagen may anchor the middle deltoid to the superior acromion and prevent retraction.
\end{abstract}

$\mathbf{R}$ otator cuff (RC) pathology is common, yet the pathogenesis remains incompletely understood. The morphology of the scapula and acromion have recently been proposed to play a role in the development of RC tears by altering the biomechanics of the shoulder and, hence, leading to intrinsic degeneration of the RC tendons. The critical shoulder angle (CSA) (defined as the angle between a line connecting the superior and inferior aspects of the glenoid fossa and another line

From the University of New South Wales (G.C.S.S., V.K.L., P.H.L.), Sydney; St George Orthopaedic Department (G.C.S.S.), Sydney; Orthopaedic Research Institute (P.H.L.), Sydney; and Douglass Hanly Moir Pathology (S.F.B.), Sydney, New South Wales, Australia.

The authors report no conflicts of interest in the authorship and publication of this article. Full ICMJE author disclosure forms are available for this article online, as supplementary material.

Received January 23, 2020; accepted June 8, 2020.

Address correspondence to Geoffrey C.S. Smith, M.B.B.Ch., F.R.A.C.S., Suite 5, Level 2, 19 Kensington Street, Kogarah, New South Wales 2217, Australia.E-mail:admin@drgeoffreysmith.com.au

(C) 2020 THE AUTHORS. Published by Elsevier Inc. on behalf of the Arthroscopy Association of North America. This is an open access article under the CC BY-NC-ND license (http://creativecommons.org/licenses/by-nc-nd/4.0/). 2666-061X/191414

https://doi.org/10.1016/j.asmr.2020.06.014 connecting the inferior aspect of the glenoid with the most inferolateral point on the acromion-the critical acromion point) has been linked to both osteoarthritis of the glenohumeral joint and RC tears ${ }^{1-3}$ (Fig 1A, B).

CSAs above $35^{\circ}$ (normal range $30^{\circ}-35^{\circ}$ ) are associated with an increased risk of RC tear, which is thought to occur through altered deltoid biomechanics. ${ }^{4-10} \mathrm{~A}$ high CSA results in a more vertical deltoid force vector through 2 mechanisms: (1) the longer acromion increases the lever arm of the deltoid, increasing its mechanical pull; (2) a larger glenoid inclination reduces the vertical deltoid force required to cause proximal migration of the humerus. ${ }^{11-13}$ In the face of an increased vertical force vector, a larger compressive force is required by the RC to restore shoulder stability and prevent proximal humeral migration. It is hypothesized that chronic overload and overuse of the supraspinatus could then predispose to an RC tear. ${ }^{14}$

A reduction of a high CSA into the normal range $\left(30^{\circ}-35^{\circ}\right)$ would theoretically reduce the vertical component of the deltoid force vector. This would decrease the mechanical load required by the supraspinatus muscle to maintain shoulder stability and possibly reduce an individual's risk of an RC tear. A lateral acromioplasty aiming to remove the most lateral part of 




the acromion and, thereby, lower the CSA has been described. ${ }^{15-17}$ The technique and orientation of the lateral acromioplasty is often not described completely; however, in 1 study, the authors described a vertical resection of the entire lateral wall of the acromion perpendicular to the undersurface of the acromion. ${ }^{17}$ However, the middle deltoid originates from the lateral acromion, and a vertical resection may, therefore, release its origin. Two previous cadaveric studies investigating the safety of a lateral acromioplasty found no damage to the inner deltoid sheath or the deltoid origin after an arthroscopic lateral acromioplasty. ${ }^{15,16}$ These investigations, however, did not examine the microscopic changes that occur at the middle deltoid origin after acromioplasty and may have involved a less aggressive lateral beveling of the undersurface of the acromion, which would likely endanger less of the deltoid origin. ${ }^{15}$ In spite of the potential to release more of the middle deltoid origin, the more aggressive vertical resection has demonstrated good outcomes and no evidence of retraction of the deltoid on follow-up MRI studies. ${ }^{17}$

The aim of this study was to assess the nature of the middle deltoid muscle insertion onto the lateral acromion by macroscopic, MRI and histologic examination and to, thereby, assess the potential impact of a vertical lateral acromioplasty on the deltoid origin. We hypothesized that the middle deltoid muscle attaches to the entire lateral acromion.

\section{Methods}

Six fresh-frozen cadaveric shoulders were obtained (mean age 68 years, range 56-81). Ethics approval for the project (HC \#HC17694) was obtained on February 28, 2018, from the Human Research Advisory Panel, University of New South Wales, Australia. The shoulders

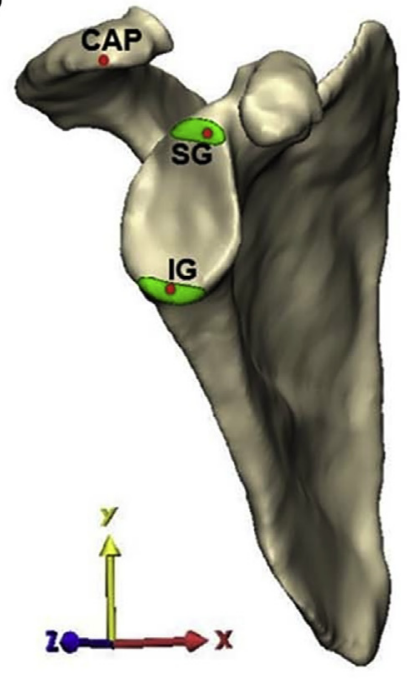

Fig 1. (A) The critical shoulder angle (CSA) was originally measured on AP shoulder radiographs. (B) The critical acromion point (CAP) is the most lateral point on the acromion and corresponds to the acromial landmark in measuring the CSA. (Karns MR, Jacxsens M, Uffmann WJ, e al. The critical acromial point: The anatomic location of the lateral acromion in the critical shoulder angle. $J$ Shoulder Elbow Surg 2018;27:151-159). Reprinted with permission. underwent a 3T MRI scan with $\mathrm{Tl}$ and proton density (PD) sequences with fat suppression. The nature of the deltoid origin from the lateral acromion was assessed on GE MediaViewer (GE Healthcare, Chicago, IL, USA) by a fellowship-trained shoulder surgeon. The thickness of the muscle origin and the superficial deltoid fascia were measured on coronal PD-weighted images at the level of the critical acromion point (CAP) using digital calipers. The CAP was identified on the coronal images as the most inferolateral point of the acromion.

The scapulae were osteotomized vertically at the suprascapular notch, and the acromion was fixed onto a cement block. The skin, subcutaneous tissue and superficial layers of fascia were dissected and removed. The deltoid was released from the humerus distally and then sectioned through the muscle $2 \mathrm{~cm}$ distal to its origin from the scapula. All soft-tissue attachments to the glenoid were removed, and the humerus was removed by dissecting and detaching the RC tendons. The deltoid muscle origin and the coracoacromial ligament were preserved (Fig 2).

The CAP, defined as a tubercle that is the most lateral point on the acromion, was identified by palpation by 2 independent observers. ${ }^{3}$ The anteroposterior length of the acromion and the distance to the CAP was measured using digital calipers (Mitutoyo, Kanagawa, Japan). The nature and location of the middle deltoid origin from the lateral acromion was assessed macroscopically, viewing it from both the superior and the inferior aspects.

Photographs were taken of the acromions in an Al orientation according to the Suter-Henninger criteria (defined as the overlapping borders of the anterior and posterior glenoid rim and the coracoid process intersecting the upper edge of the glenoid face) to ensure 


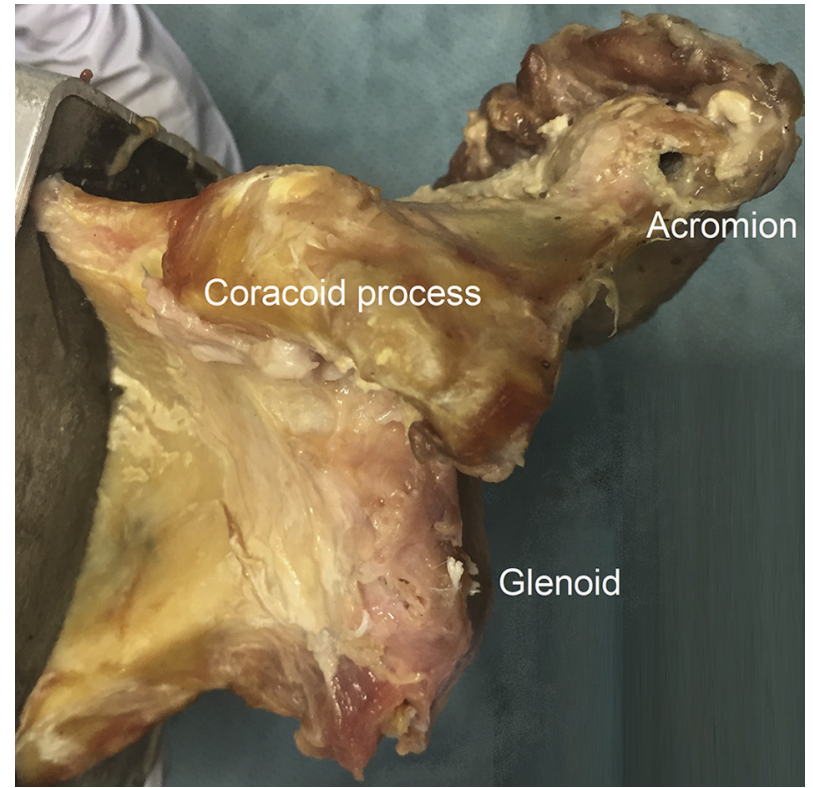

Fig 2. Preparation of a left shoulder cadaver by dissecting away tissues to reveal the glenoid. Deep soft-tissue attachments to the acromion were preserved; superficial tissues were dissected.

accurate measurement of the CSA. ${ }^{18}$ This was performed using a modification of the technique described by Moor et al. ${ }^{1}$ (for true anteroposterior radiographs) by using photographs rather than anteroposterior radiographs; 2 independent observers used ImageJ (U.S. National Institutes of Health, Bethesda, MD, USA) (Fig 3). ${ }^{19}$ Intraobserver reliability and interobserver reliability (ICC values) were assessed by a l-way random model on IBM SPSS Statistics (version 25, SPSS, Chicago, IL, USA).

Ten-mm blocks were then taken from the acromion in the coronal plane at the CAP, and at $1 \mathrm{~cm}$ and $2 \mathrm{~cm}$ posterior to the CAP (Fig 4). The CAP is a relevant landmark in a lateral acromioplasty because the most lateral portion of bone (the CAP) would be removed first. The specimens were fixed in $10 \%$ buffered formalin, serially sectioned from anterior to posterior, decalcified in rugged disolved oxygen, and embedded in paraffin. Then, $5 \mu \mathrm{m}$ sections were stained with H\&E and Masson trichrome. The enthesis of the deltoid origin was examined under light and polarized light microscopy (Olympus BX51-P, Shinjuku, Tokyo) and reviewed by a musculoskeletal pathologist. The thickness of the muscle origin and the superficial deltoid fascia were measured on the Masson trichome-stained specimens at the CAP.

\section{Results}

The mean anteroposterior length of the acromion specimens was $50.4 \mathrm{~mm}$ (range 47.6-52.9 $\mathrm{mm}$ ). The distance from the anterior edge of the acromion to the
CAP was a mean of $21.9 \mathrm{~mm}$ (range 17.7-23.8 mm), and the thickness of the acromion at the CAP was $10.5 \mathrm{~mm}$ (range 8.77-11.47 $\mathrm{mm}$ ). The mean photographic CSA was $34.92^{\circ} \pm 2.91^{\circ}$ (Table 1 ). Intraobserver reliability for photographic CSA measurements was excellent, with an ICC of 0.94 (95\% CI 0.69-0.99) and 0.93 (95\% CI 0.63 0.99 ) for both observers. Interobserver reliability was also excellent, with an ICC of 0.95 (95\% CI 0.73-0.99). Macroscopic inspection of the deltoid origin revealed that the deltoid origin occupied the entire vertical extent of the lateral border of the acromion in all specimens. Some fibrous tissue extended medially from the superior surface of the deltoid onto the superior surface of the acromion in all cases.

Examination of coronal Tl- and PD-weighted MRI scans also demonstrated that the middle deltoid muscle inserted onto the entire lateral wall of the acromion. On all the PD-weighted images, there was an area of low signal intensity at the superior aspect of the lateral margin of the acromion. This thinned and blended with the superficial surface of the deltoid further laterally (Table 2).

On the corresponding histology slides, the Masson trichrome stains were used to obtain a microscopic view of the middle deltoid origin. The enthesis of the deltoid muscle consisted of dense birefringent bundles of collagen when viewed under polarized microscopy (Fig 5). This collagen inserted onto the endplate of the lateral acromion through a narrow zone of fibrocartilaginous tissue with chondrocyte-like cells, often noted at entheses. The bony endplate of the acromion was

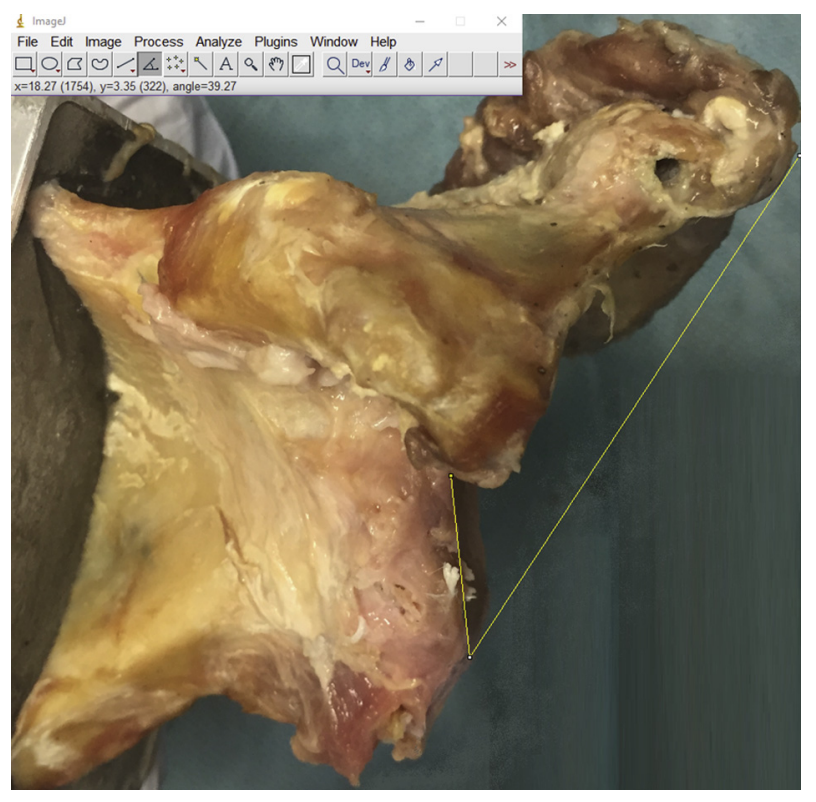

Fig 3. A photograph of a left shoulder positioned in the Al orientation according to the Suter-Heninger classification. The critical shoulder angle degrees being measured by ImageJ using the technique described by Moor et al. ${ }^{1}$ 


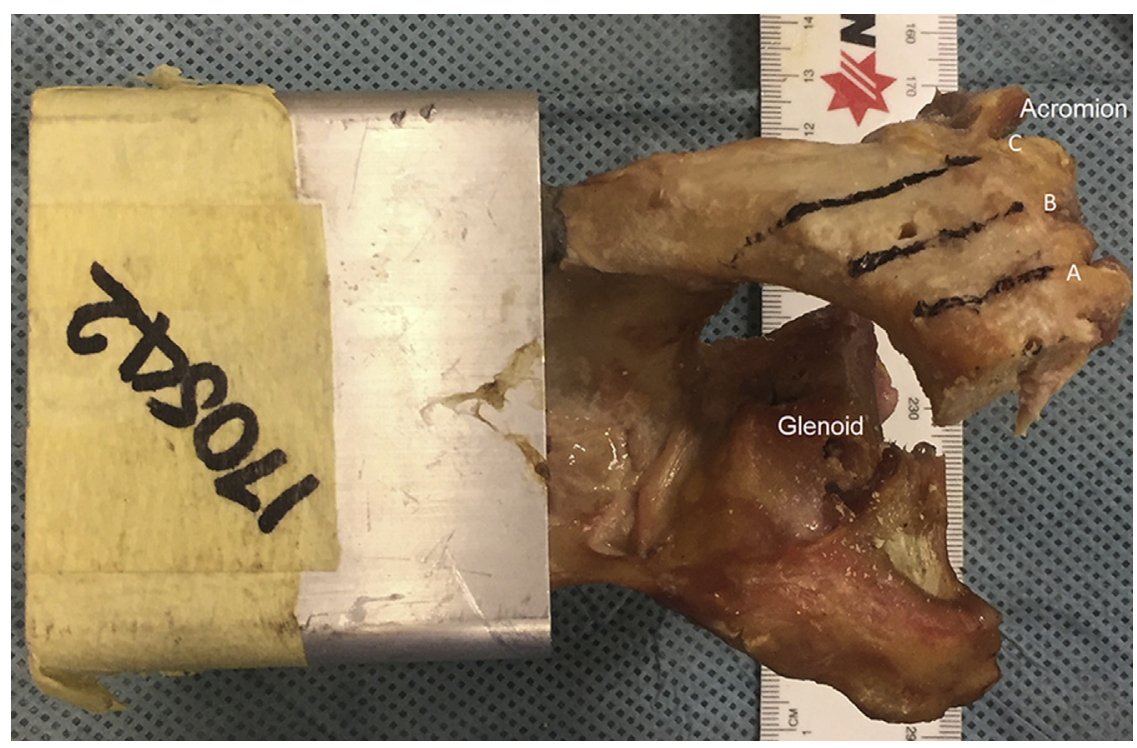

Fig 4. Preparing a left-shoulder specimen for histologic analysis. Line A, a coronal section was taken through the critical acromion point (CAP). Lines B and C, coronal sections were taken $1 \mathrm{~cm}$ and $2 \mathrm{~cm}$ posterior to the CAP, respectively.

composed of a continuous layer of mature lamellar bone.

The birefringent collagen fibers blended with the endplate at all points on the lateral wall of the acromion; thus, the enthesis of the middle deltoid occupied the entire lateral acromion (Fig 5A), which is consistent with the findings seen on MRI (Fig 5B) and macroscopically. This was observed on all specimens, at each of the 3 sites where sections were taken. The tendinous origin of the deltoid was continuous, with organized bands of collagen that extended medially across the superior surface of the acromion (Fig 5A). These bands of collagen were seen more prominently on some specimens than on others. The superior band of collagen also extended laterally beyond the edge of the acromion and blended with the superficial deltoid fascia beyond the tendinous origin, which corresponded with the bands of low signal intensity seen on the MRI images.

\section{Discussion}

The results of this study show that the middle deltoid originates from the entire vertical extent of the lateral wall of the acromion. This suggests that a vertical lateral acromioplasty is not able to decrease the CSA without releasing the entire deltoid muscle, no matter how much bone has been resected mediolaterally. However, a more obliquely oriented beveling of the lateral acromion would partially release the middle deltoid. Further studies, including a histologic examination of the deltoid insertion after arthroscopic lateral acromioplasty, are required to confirm this conclusion.

A large CSA $(>35 \underline{0})$ has been associated with an increased risk of RC tears. It has also been linked to poorer patient-reported outcomes and an increased risk of rotator cuff retear after a repair. ${ }^{20}$ The mechanism linking an elevated or higher CSA to RC tears is an increase in the vertical force vector from the middle deltoid during shoulder abduction. A lateral acromioplasty that spares the middle deltoid origin would not alter this force vector. However, if lateral impingement is a cause of RC tears, then lateral acromioplasty could reduce the risk of $\mathrm{RC}$ tears without requiring any change in deltoid biomechanics. A vertical resection would, by necessity, release the entire middle deltoid and, therefore, the biomechanics of the deltoid would be altered. In contrast, an oblique resection that leaves some of the deltoid origin still attached to the lateral wall of the acromion would reduce the CSA and would spare some of the deltoid origin. This would mean that the biomechanics of the deltoid would be less significantly changed.

A lateral spur emanating from the lateral wall of the acromion may affect the measurement of the CSA. The original description of the CSA measurement did not specify whether a spur in the region should be included in the measurement. A spur is unlikely to have any deltoid fibers attached to it and, therefore, could be safely resected. Although, again, if this were to be the case, then there would be no effect on the biomechanics of the deltoid.

Table 1. Photographic CSA Measurements

\begin{tabular}{lc}
\hline Specimen Number & Mean CSA (photograph) \\
\hline 170507 & $32.16^{\circ}$ \\
170527 & $33.06^{\circ}$ \\
170542 & $37.79^{\circ}$ \\
170670 & $31.70^{\circ}$ \\
170681 & $37.85^{\circ}$ \\
520515 & $36.94^{\circ}$ \\
\hline
\end{tabular}

CSA, critical shoulder angle. 
Table 2. Nature of the Tissue Seen Radiologically and Histologically at the Lateral Margin of the Acromion

\begin{tabular}{|c|c|c|c|c|}
\hline \multirow[b]{2}{*}{$\begin{array}{l}\text { Specimen } \\
\text { Number }\end{array}$} & \multicolumn{2}{|c|}{ MRI } & \multicolumn{2}{|c|}{ Histology } \\
\hline & $\begin{array}{l}\text { Mean thickness of } \\
\text { fibrous tissue }(\mathrm{mm})\end{array}$ & $\begin{array}{l}\text { Mean thickness of } \\
\text { deltoid origin }(\mathrm{mm})\end{array}$ & $\begin{array}{l}\text { Mean thickness of } \\
\text { fibrous tissue }(\mathrm{mm})\end{array}$ & $\begin{array}{c}\text { Mean thickness } \\
\text { of deltoid origin }(\mathrm{mm})\end{array}$ \\
\hline 170527 & 1.6 & 5.4 & 1.0 & 6.3 \\
\hline 170542 & 0.9 & 5.4 & 0.6 & 7.4 \\
\hline 170670 & 1.1 & 4.6 & 2.0 & 6.5 \\
\hline 170681 & 1.2 & 3.4 & 0.8 & 5.9 \\
\hline
\end{tabular}

MRI, magnetic resonance imaging.

The previous cadaveric studies investigating the safety of a lateral acromioplasty found no macroscopic evidence of deltoid origin injury postacromioplasty. ${ }^{15,16}$ The middle deltoid muscle was still attached to the acromion, and the surrounding connective tissues were well preserved. In addition, there was no statistically significant difference in the pull-to-failure loads of the middle deltoid before and after lateral acromioplasty, suggesting that the mechanical integrity of middle deltoid was preserved..$^{21}$ These studies assessed the deltoid macroscopically and offered no explanation for the preservation of the deltoid origin, but in 2 of the studies, a beveling of the inferior margin of the lateral border of the acromion is described; according to our results, this would result in a partial release of the deltoid. ${ }^{15,21}$ Additionally, examination of the enthesis was limited to the superficial layers of soft-tissue attachments; thus, damage to the deeper muscle attachment could not be excluded. ${ }^{15,16,21}$

The superior band of collagen that spans from the superficial deltoid fascia to the superior aspect of the acromion could play a role in maintaining the deltoid attachment to the acromion and its mechanical strength after a lateral acromioplasty. We hypothesize that this band of collagen anchors the middle deltoid to the superior surface of the acromion when its attachment to the lateral wall is removed during a lateral acromioplasty. This could explain why previous studies have shown no macroscopic injury to the deltoid after lateral acromioplasty, if the specimens were viewed from above, and that no evidence of deltoid retraction or fatty infiltration of the deltoid has been shown on MRI scans after a vertical lateral acromioplasty. ${ }^{17}$ Scar tissue at the deltoid origin is often seen on postoperative MRIs; it is indicative of at least some deltoid muscle injury, and it is possible that this subsequently restores the connection between the new lateral acromion edge and the middle deltoid origin. ${ }^{15-17}$ When reporting the clinical results of lateral acromioplasty, we recommend that authors describe their technique accurately in terms of either a vertical resection or an oblique undersurface beveling.
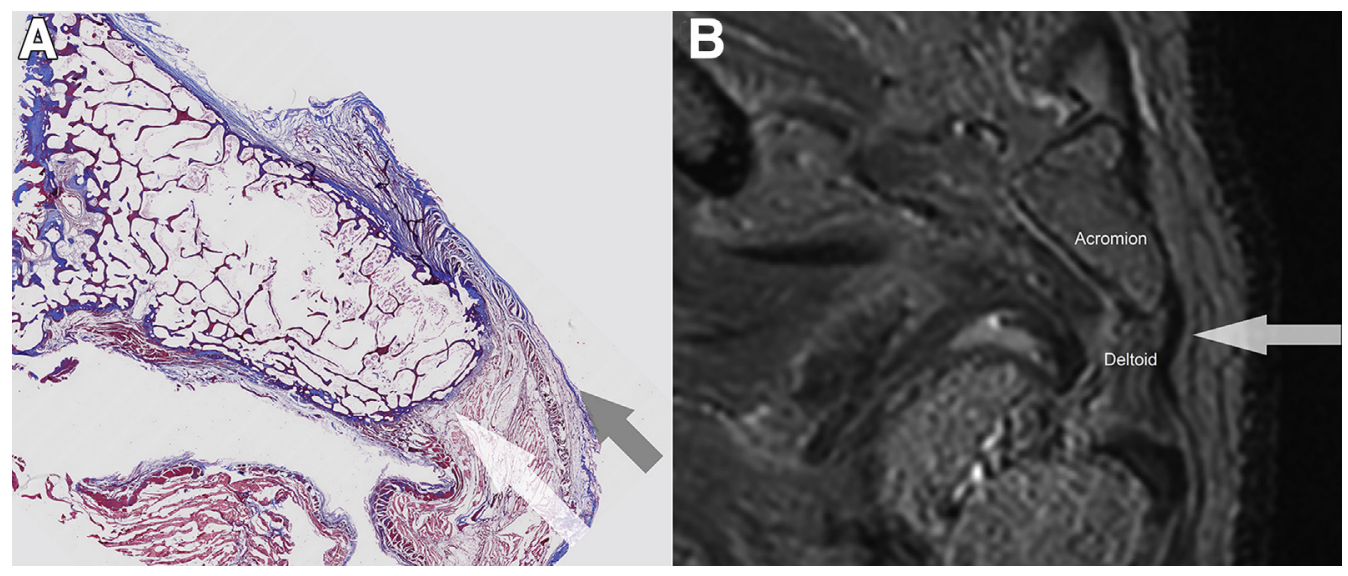

Fig 5. (A) Masson trichrome stain of a coronal acromion section. The red deltoid muscle becomes tendon, which inserts onto the endplate (blue) through a zone of fibrocartilage. The bone of the acromion appears as red. The deltoid origin (white arrow) occupies $100 \%$ of the lateral wall of the acromion. The superior band of collagen (black arrow) is part of the same fibrocartilaginous structure that covers the lateral wall of the acromion. (B) Low-intensity signal (white arrow) on proton density-weighted coronal MRI corresponds to this band of collagen, which is seen arising from the superior acromion and continuing beyond the lateral border of the deltoid. 
The main advantages of our study relate to the use of MRI and histologic examination of the deltoid origin rather than relying on macroscopic examination alone. This provided more detailed information about the deltoid origin and the potential effect of a lateral acromioplasty.

\section{Limitations}

There are limitations to consider when interpreting our study's results. The sample size was small. Our study did not consider oblique resections of the lateral acromion, which would have released only part of the deltoid origin. We have also used a photographic method to measure the CSA. This method has not been described previously, but it does allow precise alignment of the scapula according to the Suter-Henninger criteria. The good inter- and intraobserver reliability suggests that this is an accurate and reproducible method; however, it is possible that the overlying glenoid cartilage may have affected CSA measurements, though this would not have influenced the described effect of the lateral acromioplasty on the deltoid origin. This method was selected because the alternative of using the MRI technique has been shown to be inferior. ${ }^{22}$

\section{Conclusions}

The middle deltoid muscle occupies the entire lateral acromion.

\section{References}

1. Moor BK, Bouaicha S, Rothenfluh DA, et al. Is there an association between the individual anatomy of the scapula and the development of rotator cuff tears or osteoarthritis of the glenohumeral joint? A radiological study of the critical shoulder angle. Bone Joint J 2013;95-B:935-941.

2. Via AG, De Cupis M, Spoliti M, Oliva F. Clinical and biological aspects of rotator cuff tears. Muscles Ligaments Tendons J 2013;3:70-79.

3. Karns MR, Jacxsens M, Uffmann WJ, et al. The critical acromial point: The anatomic location of the lateral acromion in the critical shoulder angle. J Shoulder Elbow Surg 2018;27:151-159.

4. Chalmers PN, Salazar D, Steger-May K, et al. Does the Critical shoulder angle correlate with rotator cuff tear progression? Clin Orthopaed Relat Res 2017;475:1608-1617.

5. Blonna D, Giani A, Bellato E, et al. Predominance of the critical shoulder angle in the pathogenesis of degenerative diseases of the shoulder. J Shoulder Elbow Surg 2016;25: 1328-1336.

6. Cherchi L, Ciornohac JF, Godet J, et al. Critical shoulder angle: Measurement reproducibility and correlation with rotator cuff tendon tears. Orthopaed Traumatol Surg Res 2016;102:559-562.

7. Gomide LC, Carmo TCD, Bergo GHM, et al. Relationship between the critical shoulder angle and the development of rotator cuff lesions: A retrospective epidemiological study. Rev Bras Ortop 2017;52:423-427.

8. Miswan MF, Saman MS, Hui TS, et al. Correlation between anatomy of the scapula and the incidence of rotator cuff tear and glenohumeral osteoarthritis via radiological study. J Orthopaed Surg 2017;25:2309499017690317.

9. Shinagawa K, Hatta T, Yamamoto N, et al. Critical shoulder angle in an East Asian population: Correlation to the incidence of rotator cuff tear and glenohumeral osteoarthritis. J Shoulder Elbow Surg 2018;27: 1602-1606.

10. Pandey V, Vijayan D, Tapashetti S, et al. Does scapular morphology affect the integrity of the rotator cuff? J Shoulder Elbow Surg 2016;25:413-421.

11. Nyffeler RW, Werner CM, Sukthankar A, et al. Association of a large lateral extension of the acromion with rotator cuff tears. J Bone Joint Surg 2006;88:800-805.

12. Gerber C, Snedeker JG, Baumgartner D, Viehofer AF. Supraspinatus tendon load during abduction is dependent on the size of the critical shoulder angle: A biomechanical analysis. J Orthopaed Res 2014;32:952-957.

13. Wong AS, Gallo L, Kuhn JE, et al. The effect of glenoid inclination on superior humeral head migration. J Shoulder Elbow Surg 2003;12:360-364.

14. Viehofer AF, Gerber C, Favre P, et al. A larger critical shoulder angle requires more rotator cuff activity to preserve joint stability. J Orthopaed Res 2016;34:961-968.

15. Katthagen JC, Marchetti DC, Tahal DS, et al. The effects of arthroscopic lateral acromioplasty on the critical shoulder angle and the anterolateral deltoid origin: An anatomic cadaveric study. Arthroscopy 2016;32:569-575.

16. Altintas B, Kaab M, Greiner S. Arthroscopic lateral acromion resection (ALAR) optimizes rotator cuff tear relevant scapula parameters. Arch Orthopaed Trauma Surg 2016;136:799-804.

17. Gerber C, Catanzaro S, Betz M, Ernstbrunner L. Arthroscopic correction of the critical shoulder angle through lateral acromioplasty: A safe adjunct to rotator cuff repair. Arthroscopy 2018;34:771-780.

18. Suter T, Gerber Popp A, Zhang Y, et al. The influence of radiographic viewing perspective and demographics on the critical shoulder angle. J Shoulder Elbow Surg 2015;24: el49-el58.

19. Rasband WS. ImageJ. Bestheda, Maryland, USA. 19972018.

20. Garcia GH, Liu JN, Degen RM, et al. Higher critical shoulder angle increases the risk of retear after rotator cuff repair. J Shoulder Elbow Surg 2017;26:241-245.

21. Marchetti DC, Katthagen JC, Mikula JD, et al. Impact of arthroscopic lateral acromioplasty on the mechanical and structural integrity of the lateral deltoid origin: A cadaveric study. Arthroscopy 2017;33:511-517.

22. Spiegl UJ, Horan MP, Smith SW, et al. The critical shoulder angle is associated with rotator cuff tears and shoulder osteoarthritis and is better assessed with radiographs over MRI. Knee Surg Sports Traumatol Arthrosc 2016;24:2244-2251. 